\title{
Production of tailor-made fructans in sugar beet by expression of onion fructosyltransferase genes
}

Guy Weyens ${ }^{1} \star *$, Tita Ritsema ${ }^{2}$, Kees Van Dun ${ }^{1} \dagger$, Diederick Meyer ${ }^{3}$, Murielle Lommel $^{1}$, Jean Lathouwers ${ }^{1}$, Inge Rosquin ${ }^{1}$, Pascale Denys ${ }^{1}$, Alain Tossens ${ }^{1}$, Marleen Nijs ${ }^{1}$, Stefan Turk², Nathalie Gerrits², Sjak Bink Bas Walraven ${ }^{3}$, Marc Lefèbvre ${ }^{1}$ and Sjef Smeekens ${ }^{2}$

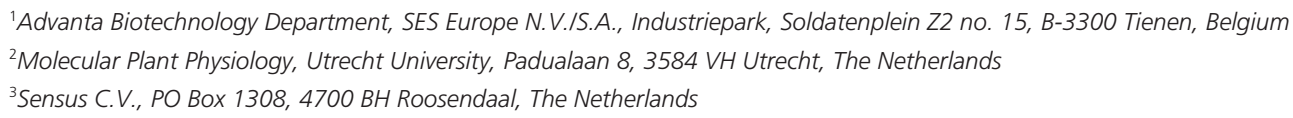

Received 1 December 2003; revised 9 February 2004; accepted 10 February 2004.

*Correspondence (fax +32 16 808263; e-mail guy.weyens@ses-europe.be) tPresent address: Rijk Zwaan Nederland BV, Postbus 40, 2678 ZG De Lier, The Netherlands

Keywords: carbohydrate, fructan, fructosyltransferase, inulin neo-series, onion, sugar beet.

\begin{abstract}
Summary
The consumption of fructans as a low caloric food ingredient or dietary fibre is rapidly increasing due to health benefits. Presently, the most important fructan source is chicory, but these fructans have a simple linear structure and are prone to degradation. Additional sources of high-quality tailor-made fructans would provide novel opportunities for their use as food ingredients. Sugar beet is a highly productive crop that does not normally synthesize fructans. We have introduced specific onion fructosyltransferases into sugar beet. This resulted in an efficient conversion of sucrose into complex, onion-type fructans, without the loss of storage carbohydrate content.
\end{abstract}

\section{Introduction}

Food offering health benefits is much in demand nowadays. One category of such food is called functional food. Functional food has acknowledged health benefits and/or disease preventive abilities and is a common part of the daily diet (Roberfroid, 1999). Fructans (polyfructosylsucroses) are one of the most promising ingredients for functional foods to date; they are present in a normal diet and possess widely recognized benefits for health (Ritsema and Smeekens, 2003). Fructans are considered to be prebiotics as they selectively promote the growth of beneficial intestinal bacteria, such as the resident bifidobacteria (Rao, 1999). Furthermore, fructans prevent the formation of aberrant crypt foci (early indication of colon cancer), promote mineral resorption, decrease cholesterol levels and decrease insulin levels (Kaur and Gupta, 2002). Therefore, fructans have been claimed to have favourable effects in the prevention of cardiovascular diseases, colon cancer and osteoporosis.

Humans lack the ability to metabolize fructans. Therefore, fructans are also classified as low caloric food ingredients, replacing sugar (short-chain inulin) and/or fat (longer chain inulin).

The inulin-type fructans, which are commercially available, have been used mostly to study health effects. However, recently, it has been reported that neokestose is a superior growth promoter of bifidobacteria than short-chain inulin (Kilian et al., 2002).

To further take advantage of fructans as health-promoting molecules and to meet the increasing demand for different fructan structures, a more cost-effective production of tailormade fructans is needed.

Fructans are synthesized in several plants and are known for their important role as a carbohydrate reserve. They are synthesized in many economically important orders of Asterales (chicory, Jerusalem artichoke), Liliales (onion, tulip) and Poales (barley, wheat).

Fructan synthesis takes place in the vacuole (Wagner et al., 1983 ) and is initiated by sucrose:sucrose 1 -fructosyltransferase (1-SST) (Lüscher et al., 1996; Pollock and Cairns, 1991), which catalyses the fructosyl transfer from one sucrose molecule to another, resulting in the trisaccharide 1-kestose (1-kestotriose). In 1-kestose, the additional fructose moiety is coupled to the fructose residue of sucrose via a $\beta(1-2)$ linkage. 1-Kestose is used by additional fructosyltransferases to build longer and/or more complex fructans (Ritsema and Smeekens, 2003). The chain length and the linkage type of fructans depend on the kestose-using enzyme. Fructan:fructan 1-fructosyltransferase (1-FFT) elongates 1-kestose with 
additional $\beta(1-2)$-linked fructoses, producing inulin (Koops and Jonker, 1996; Van den Ende and Van Laere, 1996). In the presence of fructan:fructan $6 \mathrm{G}$-fructosyltransferase (6G-FFT), neo-kestose (6G-kestotriose) can be formed by the transfer of a fructose residue of 1-kestose to the carbon 6 of the glucose moiety of sucrose (Shiomi, 1981; Vijn et al., 1997). Neokestose can be elongated by $6 \mathrm{G}-\mathrm{FFT}$ on the two terminal fructose residues, leading to the fructan inulin neo-series (Ritsema et al., 2003).

Previously, it has been shown that the expression of fructosyltransferase genes in plants that do not accumulate fructans leads to the synthesis of fructans (Hellwege et al., 2000; Sévenier et al., 1998). Sugar beet is an economically important plant that lacks the enzymes to produce fructans, but accumulates high levels of the substrate sucrose. Therefore, we introduced a pair of fructosyltransferases from onion, namely 1-SST and 6G-FFT, into sugar beet. This resulted in highlevel accumulation of onion-type fructans in the sugar beet.

\section{Results}

\section{Fructan synthesis in transgenic sugar beet}

Stomatal guard cell protoplasts isolated from sugar beet were transformed (Hall et al., 1996) with onion 1-SST (Vijn et al., 1998), alone and in combination with 6G-FFT (Vijn et al., 1997), under the control of the constitutive ubiquitin 3 promoter of Arabidopsis. Calli were selected on bialaphos and transformed sugar beet plantlets were obtained.

To identify the transgenic plants that express the fructosyltransferases, leaf material from in vitro-grown plantlets was harvested and analysed by Shodex liquid chromatography. Although fructan levels were low in leaf material, several transgenics with DP-3 values (DP-x: DP, degree of polymerization; $x$, number of monosaccharides in the chain) above $1 \mathrm{~g} / \mathrm{kg}$ fresh weight were identified, whereas, in non-transgenic controls, the DP-3 value never exceeded $0.3 \mathrm{~g} / \mathrm{kg}$ fresh weight (data not shown). Amongst the bialaphos-resistant primary transformants harbouring the 1-SST gene alone, 38 plants were found that exhibited 1-SST activity (SST series). In primary transformants containing the 1-SST gene combined with 6G-FFT, high fructan accumulation was found in 15 lines (SSG series).

The presence of the genes was confirmed by polymerase chain reaction $(P C R)$ amplification using gene-specific primers, and the copy number was determined by Southern blot analysis (data not shown). No correlation between the copy number and enzyme activity was found.

Fructan-positive transgenic plantlets were rooted and transferred to the greenhouse. The transgenic plants expressing

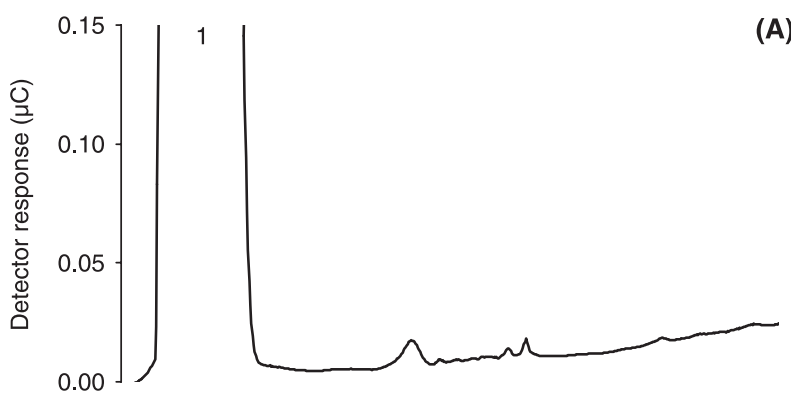

(A)
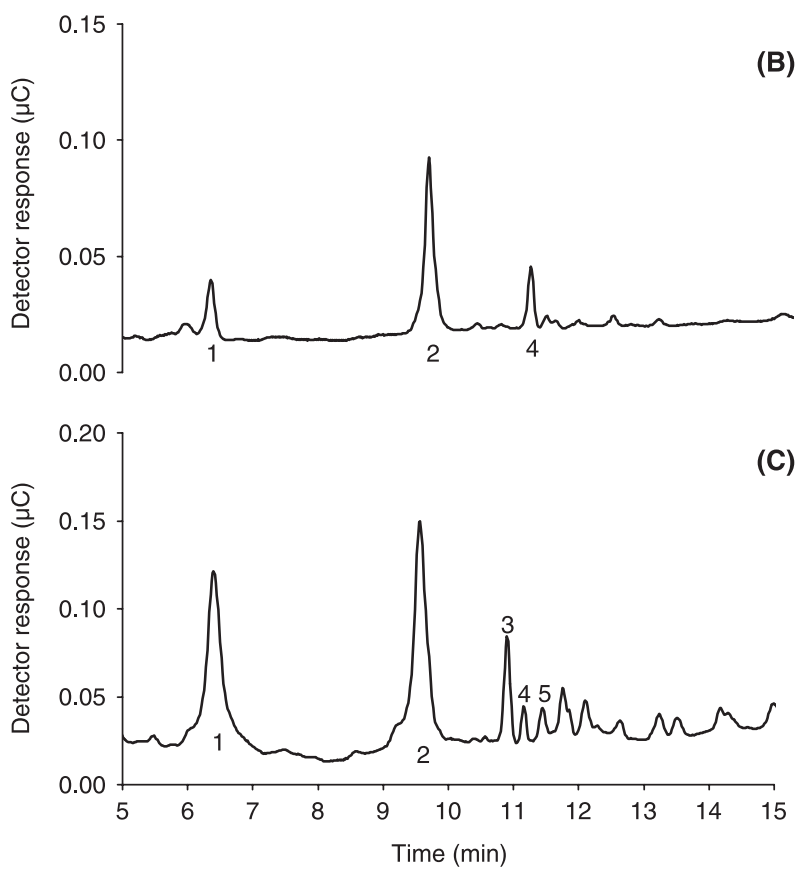

Figure 1 High-performance anion exchange chromatography (HPAEC) profile of sugars produced in an enzymatic assay of leaf extracts. Homogenized leaf material was incubated at $28^{\circ} \mathrm{C}$ overnight with sucrose at pH 5.8. (A) Wild-type. (B) SST beet. (C) SSG beet. Peaks: 1, sucrose (GF); 2, 1-kestotriose (GFF); 3, 6G-kestotriose (FGF); 4, 1,1-kestotetraose (GFFF); 5, 1 and 6G-kestotetraose (FGFF).

the 1-SST gene, alone or in combination with 6G-FFT, did not differ in overall phenotype from the non-transformed controls; taproot growth appeared to be normal under greenhouse conditions.

Leaf extracts of transgenic beets that were grown for 3 months in the greenhouse were tested for enzymatic activity. Protein extracts were incubated with sucrose, and sugar products were analysed by High-performance anion exchange chromatography (HPAEC). This analysis showed that the fructosyltransferases were active. Leaf extracts from sugar beet transformed with 1-SST produced 1-kestose, whereas leaf extracts from transgenics harbouring both 1-SST and 6G-FFT produced the inulin neo-series (Figure 1). A background enzymatic activity that produced 1-kestose was detected in non-transformed leaf material after incubation with sucrose. This is most probably due to invertase activity (Straathof et al., 1986). 
Table 1 Carbohydrate content of taproots from several sugar beet lines harvested after 3 months of growth. Carbohydrate content is represented as $\mathrm{g} / \mathrm{kg}$ fresh weight \pm standard deviation $(n=2)$

\begin{tabular}{|c|c|c|c|c|c|c|}
\hline & Fructose & Glucose & DP-2 & DP-3 & DP-4 & $D P \geq 5$ \\
\hline Control & $2.4 \pm 1.7$ & $1.2 \pm 0.8$ & $139.8 \pm 21.2$ & $3.15 \pm 1.1$ & n.d. & $0.9 \pm 0.8$ \\
\hline SST 193 & $1.7 \pm 0.2$ & $12.5 \pm 0.6$ & $39.9 \pm 0.9$ & $90.9 \pm 3.4$ & $11.4 \pm 3.4$ & $2.1 \pm 0.4$ \\
\hline SST 208 & $1.3 \pm 0.2$ & $14.2 \pm 1.9$ & $41.5 \pm 6.8$ & $88.8 \pm 11.4$ & $7.4 \pm 1.1$ & $1.6 \pm 1.2$ \\
\hline SST 406 & $0.9 \pm 0.0$ & $3.8 \pm 0.4$ & $40.7 \pm 1.6$ & $82.3 \pm 8.1$ & $5.3 \pm 1.0$ & $0.8 \pm 0.2$ \\
\hline SSG 2 & $3.9 \pm 0.4$ & $25.2 \pm 2.5$ & $65.1 \pm 4.2$ & $34.1 \pm 3.6$ & $15.0 \pm 1.3$ & $7.2 \pm 0.8$ \\
\hline SSG 25 & $1.2 \pm 0.0$ & $24.45 \pm 1.9$ & $31.1 \pm 4.0$ & $78.0 \pm 0.4$ & $7.5 \pm 1.3$ & $1.1 \pm 0.2$ \\
\hline SSG 100 & $2.3 \pm 0.2$ & $25.8 \pm 3.4$ & $36.6 \pm 3.0$ & $78.6 \pm 0.4$ & $5.9 \pm 0.2$ & $0.6 \pm 0.0$ \\
\hline SSG 134 & $3.2 \pm 1.5$ & $33.5 \pm 1.9$ & $28.7 \pm 8.7$ & $27.5 \pm 5.3$ & $19.8 \pm 1.3$ & $19.1 \pm 2.3$ \\
\hline SSG 135 & $2.4 \pm 0.0$ & $31.4 \pm 1.5$ & $45.3 \pm 2.1$ & $34.4 \pm 1.9$ & $19.5 \pm 2.1$ & $9.6 \pm 0.4$ \\
\hline SSG 175 & $1.1 \pm 0.2$ & $12.8 \pm 7.8$ & $91.1 \pm 28.6$ & $35.7 \pm 22.5$ & $2.0 \pm 1.1$ & $0.6 \pm 0.0$ \\
\hline SSG 188 & $3.0 \pm 0.0$ & $27.0 \pm 2.1$ & $46.8 \pm 0.4$ & $50.4 \pm 3.4$ & $25.7 \pm 1.9$ & $11.6 \pm 2.3$ \\
\hline
\end{tabular}

DP-x: DP, degree of polymerization; $x$, number of monosaccharides in the chain; n.d. not detected.

\section{Fructan accumulation in transgenic sugar beet roots}

Plants with high enzymatic activity in the leaf extracts were selected for further growth experiments in pots in the greenhouse, and roots were harvested after 3, 4.5 and 6 months (two roots per time point). Analysis of the root material confirmed fructan accumulation in the transgenic plants. Extractions made of only a small piece of the outer part of the sugar beet were representative for the carbohydrate content of the whole root. This was tested by comparing the data of the small-scale extractions with those of the whole taproot. This showed that both extracts gave essentially identical results (data not shown).

In root extracts of the 1-SST transformants, 1-kestose was the predominant oligosaccharide (Table 1, Figure 2). The enzymatic activity measured in leaf extracts correlated well with the DP-3 content in the roots.

Beet material from the SST 406 transformant was used in standard extraction procedures (filtration, ion exchange, etc.) as applied in industrial-scale inulin production (Hirayama and Hidaka, 1993). This process yielded a pure syrup with the carbohydrate ratios as found in the small-scale extract. The properties of this syrup were comparable with those of fructooligosaccharide preparations derived from chicory, which are currently commercially available (Table 2).

The conversion of 1-kestose into higher molecular weight fructans in the transgenic sugar beet plants was observed in plants transformed with the plasmid carrying both the 1-SST and 6G-FFT genes, indicating that the 1-kestose produced from sucrose by 1 -SST was efficiently utilized by $6 \mathrm{G}-\mathrm{FFT}$ (Table 1, Figure 2).

A comparison of the fructan concentrations at 3,4.5 and 6 months indicated that the fructan content was constant

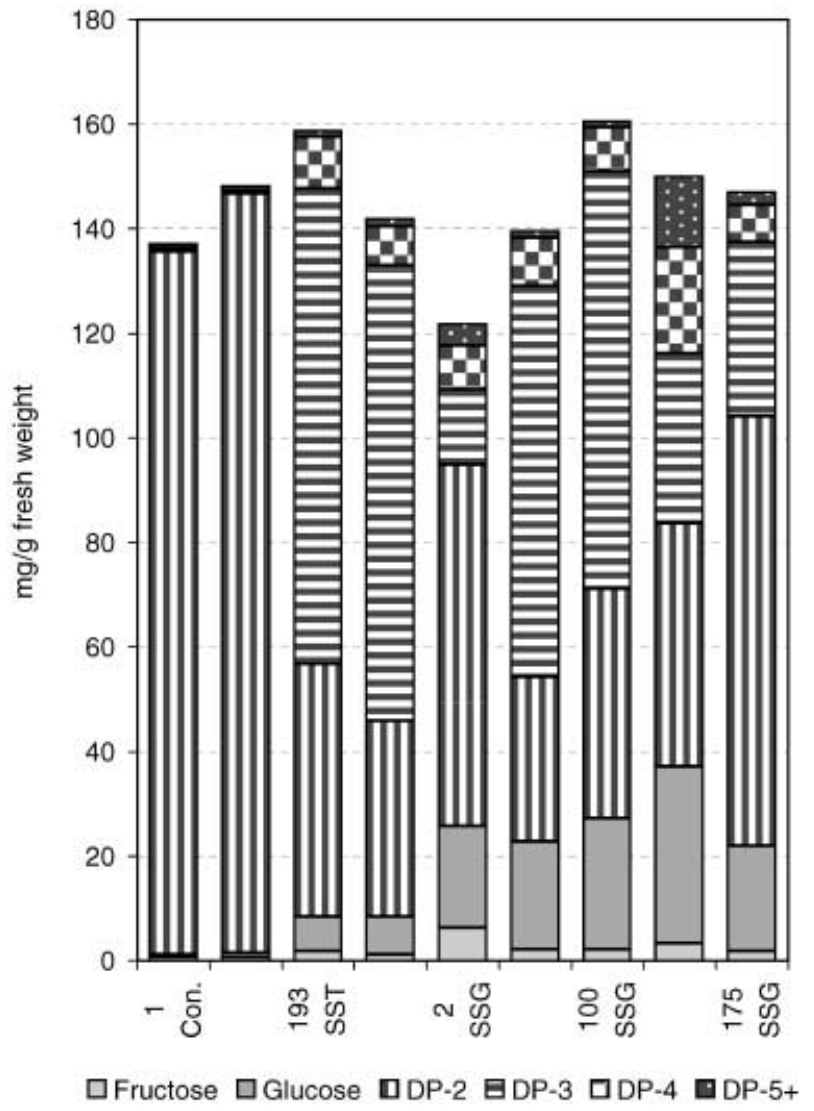

Figure 2 Carbohydrate content of taproots from different (transgenic) sugar beet lines after 6 months' growth. DP-2, sucrose; DP-3, $-4,-5+$, fructans with a length of $3,4,5$ or more, respectively.

over time (data not shown). Importantly, the total storage carbohydrate content of the transgenic beets stayed constant compared with the wild-type (Table 1, Figure 2).

HPAEC analysis showed that the elution pattern of peaks from the SSG transformants closely resembled that of onion, 
Table 2 Carbohydrate content of syrup isolated from transgenic sugar beet line SST 406 and a commercial preparation of chicory. Sugars are represented as the percentage of total soluble carbohydrates

\begin{tabular}{llllll}
\hline & \multicolumn{2}{l}{ Raw juice } & & & \multicolumn{2}{l}{ Processed syrup } \\
\cline { 5 - 6 } & SST 406 & Chicory & & SST 406 & Frutalose ${ }^{\text {TM }}$ L60 \\
& & & & & \\
\hline Fructose & 0.05 & 0.1 & & 0.8 & $6-9$ \\
Glucose & 0.2 & 0.1 & & 3.3 & \\
DP-2 & 1.7 & 0.3 & & 28.9 & $21-24$ \\
DP-3 & 2.5 & 0.2 & & 44.7 & $44-46.5$ \\
DP-4 & 0.1 & 0.2 & & 1.6 & \\
DP $\geq 5$ & 0.05 & 5.3 & 0.3 & \\
& & & & & \\
\hline
\end{tabular}

DP-x: DP, degree of polymerization; $x$, number of monosaccharides in the chain.

indicative of the presence of fructans of the inulin neo-series in beets transformed with the combination of 1-SST and 6G-FFT genes (Figure 3). This shows that the fructans normally present in onion are synthesized in sugar beet harbouring the two known fructosyltransferase genes from onion, 1-SST and 6G-FFT.

\section{Discussion}

We introduced the 1-SST cDNA from onion into sugar beet. This resulted in the accumulation of 1-kestose accompanied by a decrease in sucrose. The total storage carbohydrate content was not affected, indicating that the conversion of sucrose into fructans does not affect the basic physiological processes in the plant.

For sugar beet transformed with the 1-SST gene isolated from Helianthus tuberosus, a somewhat higher conversion of the stored sucrose into low molecular weight fructans was reported by Sévenier et al. (1998). However, this was accompanied by a decrease in overall storage carbohydrates. These differences may be due to the different expression level of the 1-SST gene, as the gene from $H$. tuberosus was driven by an enhanced 35S promoter, known for its high rate of transcription, in the study by Sévenier et al. (1998), whereas, in this study, the fructosyltransferase genes were driven by the ubiquitin 3 promoter. An enhanced activity of 1-SST may lead to the deregulation of physiological processes, resulting in a lower storage carbohydrate content.

In vitro analysis of 6G-FFT activity led to the prediction that 1-SST and 6G-FFT could produce a full array of onion fructans (Ritsema et al., 2003). Indeed, the introduction of a double construct of onion 1-SST and 6G-FFT into sugar beet resulted in vivo in a fructan profile closely resembling that from onion, indicating the efficient synthesis of neo-series inulin by these two enzymes. In addition, in the transgenic sugar beet harbouring the double construct, the total storage carbohydrate content resembled that of the wild-type, indicating that the conversion of sucrose into onion-type fructans does not affect the storage capacity.

Pilot processing of the transgenic sugar beet suggests that the fructan extraction process currently used for chicory inulin is also applicable for fructan-producing sugar beet.

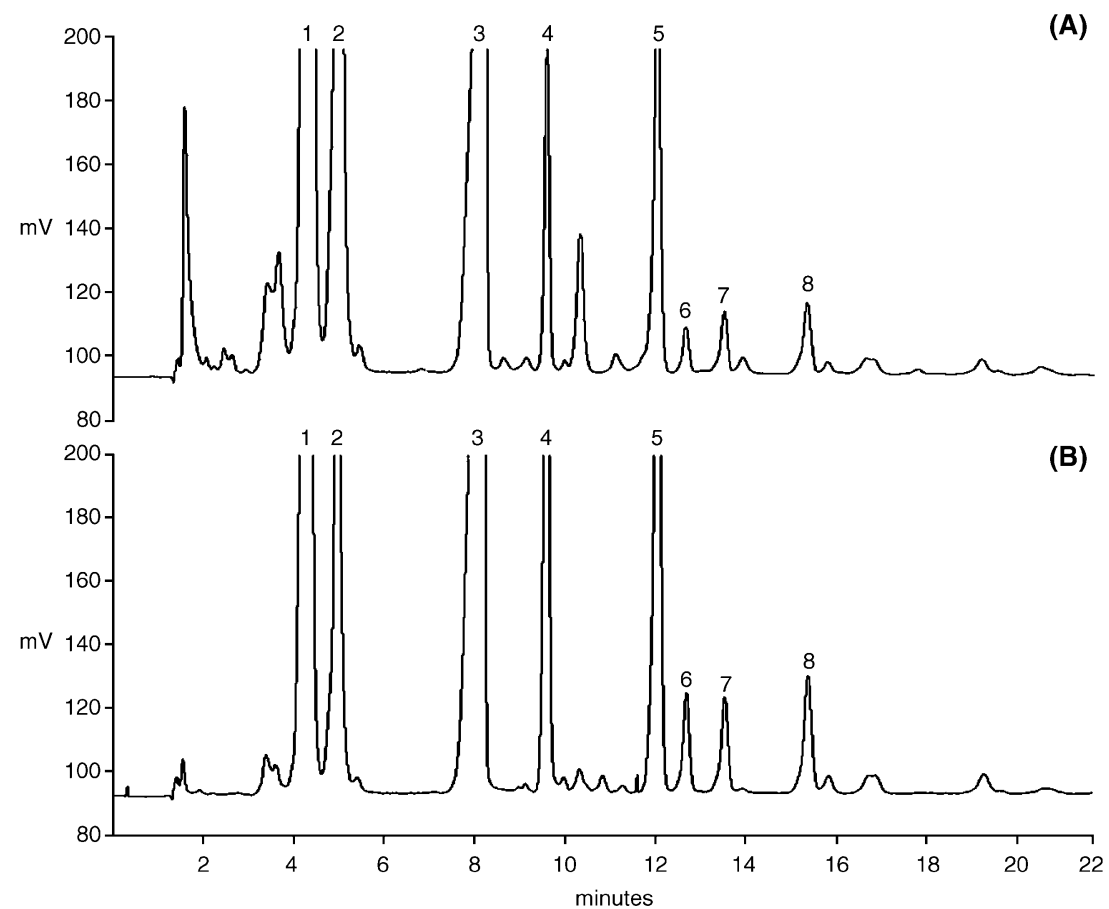

Figure 3 High-performance anion exchange chromatography (HPAEC) profile of sugars from onion (A) and transgenic sugar beet SSG 2 (B). 1, glucose; 2 , fructose; 3 , sucrose; 4, 1-kestotriose (GFF); 5, 6G-kestotriose (FGF); 6, 1,1kestotetraose (GFFF); 7, 1 and 6G-kestotetraose (FGFF); 8, 1,6G-kestotetraose (FFGF). The peak in panel $A$ between 4 and 5 is probably the breakdown product inulobiose (FF). 
Neo-series inulin has two fructose chains, one initiated at the $C 1$ carbon atom of the fructose moiety of the central sucrose and one at the C 6 position of the glucose moiety. These neo-series sugars are of interest, as neokestose ( 1 and $6 \mathrm{G}-$ kestotetraose) has been shown to stimulate the growth of bifidobacteria to a higher extent than commercially available inulin. This is indicative of the enhanced prebiotic qualities of neo-series inulin (Kilian et al., 2002).

At present, there is growing interest from the food, chemical and pharmaceutical industries to exploit the potential health benefits of fructans for humans and animals. Furthermore, in order to develop industrial products based on renewable resources with specific characteristics, several methods have been investigated to chemically modify inulin and its derivatives (Stevens et al., 2001). Potential applications include emulsifiers in cosmetics and additives in the textile and paper industry. However, the large-scale use of fructans is hindered by the production costs. Highly productive crops such as sugar beet, engineered to synthesize tailor-made fructans, are an attractive alternative. Our results demonstrate that sugar beet can be used to produce such tailor-made fructan molecules.

\section{Experimental procedures}

\section{Plasmid vectors for transformation of sugar beet}

The coding sequences of onion fructosyltransferases (Vijn et al., 1997, 1998) were inserted into a pUC19 derived vector containing the pat gene, encoding phosphinothricin acetyl transferase (Bayer Cropscience, Germany), fused to a cauliflower mosaic virus (CaMV) 355 promoter, as a selectable marker. The fructosyltransferase genes were fused to the ubiquitin 3 promoter from Arabidopsis thaliana and the 3' polyadenylation signals derived from the nopaline synthase gene of Agrobacterium tumefaciens.

\section{Sugar beet transformation}

Two diploid sugar beet breeding lines, BV-NF (pollinator) and 4D6834 (O-Type), were used. In vitro shoot cultures were initiated and maintained with a 4-week subculture period. The protocol detailed by Hall et al. (1996) was applied in all transformation experiments. The selection of transformants was started 1 week after protoplast isolation by the addition of $200 \mu \mathrm{g} / \mathrm{L}$ bialaphos. Selection was maintained during callus growth and regeneration ( $250 \mu \mathrm{g} / \mathrm{L}$ bialaphos). Transgenic shoots were subcultured on non-selective medium.

\section{Carbohydrate analysis}

The amounts of monosaccharides, disaccharides, DP-3, DP-4 and DP > 5 were determined by high-performance liquid chromatography (HPLC) using a column with gel filtration characteristics (Shodex KS-802, with a Shodex type KS-800P guard column). The mobile phase was demineralized water with a flow rate of $1 \mathrm{~mL} / \mathrm{min}$. The column temperature was $50{ }^{\circ} \mathrm{C}$ and a refractive index detector (ERMA type ERC 7512) was used at a temperature of $35-40{ }^{\circ} \mathrm{C}$.

To determine the oligofructan pattern in more detail, HPAEC was used. To that end, a Dionex DX-300 apparatus, fitted with a Dionex PA-100 column and a Dionex PA-100 guard column, was applied with Pulsed Amperometric Detection. The column temperature was $20^{\circ} \mathrm{C}$. The peaks were separated with a gradient of $\mathrm{NaOH}(0.25 \mathrm{M}) /$ sodium acetate $(0.65 \mathrm{M})$, starting with a volume ratio of $\mathrm{NaOH} /$ $\mathrm{H}_{2} \mathrm{O} /$ sodium acetate of $40 / 60 / 0$ and ending with a ratio of $1 / 39 / 60$.

Integration of the peaks obtained with Shodex or Dionex was performed using Millennium software (Waters Co.). Peak areas from the Shodex analysis were converted to quantities with the appropriate standards, whereas, for Dionex analysis, only the relative concentrations can be obtained, as standards are not available.

\section{Enzyme activity assays and sugar analysis}

In order to assay the fructosyltransferases in the transgenic sugar beet plants, leaf blades were homogenized in a dismembrator (cooled at $-80^{\circ} \mathrm{C}$ ) at 2800 r.p.m. for 1 min and then centrifuged at $15700 \mathrm{~g}$ at $4^{\circ} \mathrm{C}$ for $15 \mathrm{~min}$. To $8 \mu \mathrm{L}$ of supernatant, $1 \mu \mathrm{L}$ of sucrose solution and $1 \mu \mathrm{L}$ of $0.5 \mathrm{~m} 2-(\mathrm{N}$ Morpholino)-ethanesulfonic acid (MES) buffer were added and incubated overnight at $28^{\circ} \mathrm{C}$. For SST activity, the final sucrose concentration was $0.1 \mathrm{~m}$; for SSG, this was $0.2 \mathrm{M}$. The reaction was stopped by incubation at $95^{\circ} \mathrm{C}$ for $5 \mathrm{~min}$. The incubation mixture was diluted with $50 \mu \mathrm{L}$ of water, and $10 \mu \mathrm{L}$ was injected and analysed by HPAEC using a CarboPac PA-100 column (Dionex, Sunnyvale, CA, USA) and a water/ $\mathrm{NaOH} / \mathrm{NaAc}$ gradient. Solutions: (A) water; (B) $0.5 \mathrm{M} \mathrm{NaOH}$; and (C) $1 \mathrm{~m} \mathrm{NaAc}$. The running profile applied was as follows: $T=0$ min: $80 \%$ A, 20\% B; $T=5 \min : 50 \%$ A, 50\% B; $T=15 \min : 40 \%$ A, 50\% B, 10\% C; $T=20 \mathrm{~min}: 33 \% A$, $50 \%$ B, 17\% C; $T=35 \mathrm{~min}: 50 \%$ B, 50\% C. We observed the same peaks in onion as reported previously by Shiomi et al. (1997) and Ernst et al. (1998). We used peak identification as proposed by Ernst et al. (1998) and nomenclature as proposed by Lewis (1993) and Waterhouse and Chatterton (1993). 


\section{Extraction and characterization of fructans}

To determine the presence of fructans in freeze-dried leaves, the samples were weighed in Eppendorf tubes, mixed with twice the weight of demineralized water and incubated for $45 \mathrm{~min}$ at $70^{\circ} \mathrm{C}$. After centrifugation (15 min at $12000 \mathrm{~g}$ ), the supernatant was analysed for fructans by Shodex analysis as described above.

For the analysis of fructans in sugar beet, a small part of the outer layer of the frozen root was removed and a sample was taken from the underlying part of the root (approximately $3-$ $5 \mathrm{~g}$ of root material). The sample was squeezed through a garlic press, weighed and transferred to centrifuge tubes. Demineralized water was added at twice the weight of the sample. After mixing, the tube was placed at $70{ }^{\circ} \mathrm{C}$ (in a water bath) for 30-60 min. Finally, the sample was centrifuged for $15 \mathrm{~min}$ at $12000 \mathbf{g}$ and the supernatant was collected for the analysis of the fructan pattern (Shodex and Dionex analysis).

The fructan content of the material was determined in extracts made from selected beets. To about $100 \mathrm{~g}$ of beet, twice the weight of demineralized water was added. The mixture was homogenized in a Waring blender at maximum speed. Extraction took place at $70^{\circ} \mathrm{C}$ for $60 \mathrm{~min}$ in a water bath. Cellular debris was removed by filtration over cotton cloth. The filtrate was centrifuged for $20 \mathrm{~min}$ at $15000 \mathbf{g}$ to remove smaller debris. The clear supernatant was aspirated and adjusted to $\mathrm{pH} 4.7$ with $5 \%(\mathrm{w} / \mathrm{v}) \mathrm{H}_{2} \mathrm{SO}_{4}$. Fructozyme (Novozymes) was added at a rate of $20 \mathrm{INU} / \mathrm{g}$ fructans (the fructan content in the extract was estimated from the refractive index) and the mixture was incubated at $60^{\circ} \mathrm{C}$ for $21 \mathrm{~h}$. Samples were taken at the start and at the end and analysed for fructans with Shodex and Dionex analysis as described above. A sample without enzyme was used as the control. The Fructozyme hydrolysis further supported the fact that the fructans, as extracted, had a $\beta-2,1$ backbone.

A fructan extract of onion (Allium cepa) was made in a similar way: a sample of onions obtained at a local market was diluted with twice the weight of demineralized water and homogenized in a Waring blender. The mixture was extracted for $45 \mathrm{~min}$ at $70{ }^{\circ} \mathrm{C}$. Debris was removed by filtration and centrifugation as described above. The fructan pattern in the clear supernatant was assessed by Dionex analysis as described above.

\section{Preparation of syrup}

Beets were homogenized with 1.5 times their weight of demineralized water in a Braun blender. The homogenate was heated at $85^{\circ} \mathrm{C}$ for 90 min to extract the carbohydrates.
Debris was removed by filtration over cheese cloth. The filter cake was extracted as above with demineralized water and filtered again. The combined filtrates were centrifuged (20 min, $15000 \boldsymbol{g}$ ) and the supernatants were pooled. Protein and salts were removed by ion exchange on Bayer (Wuppertal) ion exchange resins (S100 and MP64). The carbohydratecontaining fractions were pooled and evaporated to a dry solid content of about 15\% (w/w). To remove all insoluble material, the syrup was centrifuged for $30 \mathrm{~min}$ at $15000 \mathbf{g}$. The clear supernatant was evaporated to a syrup of about $75 \%(w / w)$. The fructan content and composition were determined as described above.

\section{Acknowledgements}

Wim van der Waal and his collaborators are gratefully acknowledged for performing the many sugar analyses, and Fred van Leeuwen for his work on interpreting the Dionex chromatograms. We also thank Ivan Costermans for his technical assistance. This work was supported by funds from project FAIR CT96 1896.

\section{References}

Ernst, M., Chatterton, N.J., Harrison, P.A. and Matitschka, G. (1998) Characterization of fructan oligomers from species of the genus Allium L. J. Plant Physiol. 153, 53-60.

Hall, R.D., Riksen-Bruinsma, T., Weyens, G.J., Rosquin, I.J., Denys, P.N., Evans, I.E., Lathouwers, J.E., Lefèbvre, M.P., Dunwell, J.M. and van Tunen, A.J. (1996) A high efficiency technique for the generation of transgenic sugar beets from stomatal guard cells. Nature Biotechnol. 14, 1133-1138.

Hellwege, E.M., Czapla, S., Jahnke, A., Willmitzer, L. and Heyer, A.G. (2000) Transgenic potato (Solanum tuberosum) tubers synthesize the full spectrum of inulin molecules naturally occurring in globe artichoke (Cynara scolymus) roots. Proc. Natl. Acad. Sci. USA, 97, 8699-8704.

Hirayama, M. and Hidaka, H. (1993) Production and utilization of microbial fructans. In: Science and Technology of Fructans (Suzuki, M. and Chatterton, N.J., eds), pp. 274-317. Boca Raton, FL: CRC Press. Kaur, N. and Gupta, A.K. (2002) Applications of inulin and oligofructose in health and nutrition. J. Biosci. 27, 703-714.

Kilian, S., Kritzinger, S., Rycroft, C., Gibson, G.R. and du Preez, J. (2002) The effects of the novel bifidogenic trisaccharide, neokestose, on the human colonic microbiota. World J. Microbiol. Biotechnol. 18, 637-644.

Koops, A.J. and Jonker, H.H. (1996) Purification and characterization of the enzymes of fructan biosynthesis in tubers of Helianthus tuberosus Colombia. II. Purification of sucrose:sucrose 1-fructosyltransferase and reconstitution of fructan synthesis in vitro with purified sucrose:sucrose 1-fructosyltransferase and fructan:fructan 1-fructosyltransferase. Plant Physiol. 110, 1167-1175.

Lewis, D.H. (1993) Nomenclature and diagrammatic representation of oligomeric fructans - a paper for discussion. New Phytol. 124, 583-594. 
Lüscher, M., Erdin, C., Sprenger, N., Hochstrasser, U., Boller, U. and Wiemken, A. (1996) Inulin synthesis by a combination of purified fructosyltransferase from tubers of Helianthus tuberosus. FEBS Lett. 385, 39-42.

Pollock, C.J. and Cairns, A.J. (1991) Fructan metabolism in grasses and cereals. Annu. Rev. Plant Physiol. Plant Mol. Biol. 42, 77-101.

Rao, A.V. (1999) Dose-response effects of inulin and oligofructose on intestinal bifidogenesis effects. J. Nutr. 129, 1442S-1445S.

Ritsema, T., Joling, J. and Smeekens, S. (2003) Fructan patterns synthesized by onion fructan:fructan 6G-fructosyltransferase (6G-FFT) expressed in tobacco BY2 cells - is fructan:fructan 1fructosyltransferase (1-FFT) needed in onion? New Phytol. 160, 61-67.

Ritsema, T. and Smeekens, S. (2003) Fructans: beneficial for plants and humans. Curr. Opin. Plant Biol. 6, 223-230.

Roberfroid, M.B. (1999) What is beneficial for health? The concept of functional food. Food Chem. Toxicol. 37, 1039-1041.

Sévenier, R., Hall, R.D., van der Meer, I., Hakkert, H.J., van Tunen, A.J. and Koops, A.J. (1998) High level fructan accumulation in a transgenic sugar beet. Nature Biotechnol. 16, 843-846.

Shiomi, N. (1981) Purification and characterization of 6G-fructosyltransferase from roots of asparagus (Asparagus officinalis L.). Carbohydr. Res. 96, 281-292.

Shiomi, N., Onodera, S. and Sakai, H. (1997) Fructo-oligosaccharide content and fructosyltransferase activity during growth of onion bulbs. New Phytol. 136, 105-113.
Stevens, C.V., Meriggi, A. and Booten, K. (2001) Chemical modification of inulin, a valuable renewable resource, and its industrial applications. Biomacromolecules, 2, 1-16.

Straathof, A.J.J., Kieboom, A.P.G. and van Bekkum, H. (1986) Invertase-catalysed fructosyl transfer in concentrated solutions of sucrose. Carbohydr. Res. 146, 154-159.

Van den Ende, W. and Van Laere, A. (1996) De-novo synthesis of fructans from sucrose in vitro by a combination of two purified enzymes (sucrose:sucrose 1-fructosyltransferase and fructan:fructan 1-fructosyltransferase) from chicory roots (Cichorium intibus L.). Planta, 200, 335-342.

Vijn, I., van Dijken, A., Lüscher, M., Bos, A., Smeets, E., Weisbeek, P., Wiemken, A. and Smeekens, S. (1998) Cloning of sucrose:sucrose 1 -fructosyltransferase from onion and synthesis of structurally defined fructan molecules from sucrose. Plant Physiol. 117, 1507-1513.

Vijn, I., van Dijken, A., Sprenger, N., van Dun, K., Weisbeek, P., Wiemken, A. and Smeekens, S. (1997) Fructan of the inulin neoseries is synthesized in transgenic chicory plants (Cichorium intybus L.) harbouring onion (Allium cepa L.) fructan:fructan 6G-fructosyltransferase. Plant J. 11, 387-398.

Wagner, W., Keller, F. and Wiemken, A. (1983) Fructan metabolism in cereals: induction in leaves and compartmentation in protoplasts and vacuoles. Z. Pflanzenphysiol. 112, 359-372.

Waterhouse, A.L. and Chatterton, N.J. (1993) Glossary of fructan terms. In: Science and Technology of Fructans (Suzuki, M. and Chatterton, N.J., eds.), pp. 1-8. Boca Raton, FL: CRC Press. 